\title{
Rats With Lesions of the Hippocampus Are Impaired on the Delayed Nonmatching-to-Sample Task
}

\author{
Robert E. Clark, ${ }^{1 *}$ Alisha N. West, ${ }^{2}$ \\ Stuart M. Zola, ${ }^{1,3,4}$ and Larry R. Squire ${ }^{1,3-5}$ \\ ${ }^{1}$ Department of Psychiatry, University of California at \\ San Diego, La Jolla, California \\ ${ }^{2}$ Department of Biology, University of California at \\ San Diego, La Jolla, California \\ ${ }^{3}$ Veterans Affairs, Health Care System, San Diego, \\ California \\ ${ }^{4}$ Department of Neurosciences, University of California at \\ San Diego, La Jolla, California \\ ${ }^{5}$ Department of Psychology, University of California at \\ San Diego, La Jolla, California
}

\begin{abstract}
Rats with ibotenic acid lesions of the hippocampus $(\mathrm{H}$ IBO) were trained on the trial-unique delayed nonmatching-to-sample task (DNMS) using a short delay of $4 \mathrm{~s}$. The H-IBO group learned the nonmatching rule as quickly as control animals. However, performance was impaired on the DNMS task when the delay between the sample and choice phase was increased to 1 or $2 \mathrm{~min}$. The use of 4-s delay (probe) trials indicated that the $\mathrm{H}-\mathrm{IBO}$ animals retained the nonmatching-tosample rule throughout testing. In a second experiment, using the same groups of rats, extended training at the 1 -min delay did not ameliorate the deficit produced by $\mathrm{H}$-IBO lesions. The finding of impaired recognition memory in rats after hippocampal lesions is consistent with findings from humans and monkeys. Several methodological issues are considered that have complicated the interpretation of earlier studies of recognition memory in rats following hippocampal lesions. The capacity for recognition memory in humans, monkeys, and rodents is discussed as a straightforward example of hippocampus-dependent (declarative) memory. Hippocampus 2001;11:176-186. Published 2001 Wiley-Liss, Inc. ${ }^{+}$
\end{abstract}

KEY WORDS: ibotenic acid; object recognition; declarative memory; discrimination learning; probe trials

\section{INTRODUCTION}

In mammals, damage to a system of anatomically related structures in the medial temporal lobe impairs the formation of declarative memory (Squire,

Grant sponsor: Medical Research Service of the Department of Veterans Affairs; Grant sponsor: National Institutes of Health; Grant numbers: MH58933, MH24600, MH11154; Grant sponsor: National Alliance for Research on Schizophrenia and Depression; Grant sponsor: Metropolitan Life Foundation; Grant sponsor: Sam and Rose Stein Institute for Research on Aging.

*Correspondence to: Robert E. Clark, Department of Psychiatry 0603, University of California at San Diego School of Medicine, La Jolla, CA 92093. E-mail: clark@whoville.ucsd.edu

Accepted for publication 22 September 2000
1992). The important structures include the hippocampal region (the CA fields, dentate gyrus, and subicular complex) and the adjacent entorhinal, perirhinal, and parahippocampal cortices (Zola-Morgan and Squire, 1993). A central issue in recent studies has concerned the contribution of the hippocampus itself. It is clear that the hippocampus is essential for acquiring many spatial tasks and other tasks that depend on relational, contextual information (Eichenbaum, 1997). However, it has been less clear whether the hippocampus is also critical for the capacity to identify a recently encountered item as familiar, i.e., whether it is essential for normal recognition memory.

Studies of amnesic patients have indicated that damage restricted to the hippocampus can impair recognition memory (Zola-Morgan et al., 1986; Rempel-Clower et al., 1996; Reed and Squire, 1997; Manns and Squire, 1999). Yet, the possibility must be considered that damage has also occurred in other structures important for memory but that the neuronal dysfunction is not sufficient to progress to cell death and be detected in histopathology. Though some experimental work argues against this covert damage hypothesis (Zola-Morgan et al., 1992; Squire and Zola, 1996), the possibility is difficult to rule out entirely (Bachevalier and Meunier, 1996).

In the monkey, the benchmark test of recognition memory has been the trial-unique, delayed nonmatching-to-sample (DNMS) task. In this task, a sample object is presented and then, after a variable delay, the sample object is presented together with a new object. To receive a reward, the monkey must choose the new object. Different pairs of objects are used on each trial. To date, there have been five studies with the DNMS task following bilateral damage to the hippocampal region in adult 
animals (Zola-Morgan et al., 1992; Alvarez et al., 1995; Murray and Mishkin, 1998; Beason-Held et al. 1999; Zola et al., 2000). Of these studies, all but one (Murray and Mishkin, 1998) found that operated monkeys were impaired at recognition memory. One of the studies (Zola et al., 2000) evaluated five different groups of monkeys with ischemic, radio frequency, or ibotenic acid damage to the hippocampal region. All groups exhibited a significant impairment on the DNMS task. When data from the five groups of monkeys with hippocampal lesions were combined $(n=18)$, the operated monkeys were impaired at delays of $15 \mathrm{~s}$ and longer (Zola et al., 2000, their Fig. 9).

The DNMS task with trial-unique objects has also been adapted for the rat (Fig. 1 in Mumby et al., 1992). Although several studies have reported that damage to the hippocampus or fornix impairs performance on this task (Mumby et al., 1992, 1995; Wiig and Bilkey, 1995), other studies have failed to find an impairment (Aggleton et al., 1986; Rothblat and Kromer, 1991; Kesner et al., 1993; Mumby et al., 1996; Duva et al., 1997). Thus, the available data from rats are not in agreement.

It is also important to note that results from the DNMS task, as it is usually administered, cannot be interpreted in a straightforward way. Ordinarily, intact performance at very short delays and progressively more impaired performance at longer delays would suggest a deficit in the ability to retain information across long intervals. Indeed, in humans this pattern of performance following medial temporal lobe lesions is fundamental to current conceptions of how the hippocampus contributes to memory (Milner, 1972). However, this same pattern of performance cannot be interpreted unambiguously in the case of animals with hippocampal lesions given the DNMS task. One difficulty is that a deficit observed at a long delay could mean either that the animal cannot retain information across such a long interval, or that the animal has forgotten the nonmatching rule. This ambiguity could be resolved by occasionally presenting very-short-delay trials (probe trials) during testing at the longer delays. Good performance on the probe trials, in the face of poor performance on the long-delay trials, would provide evidence that impaired performance reflects impaired delay-dependent memory, and is not a result of having forgotten the nonmatching rule.

A second difficulty in interpreting results from the delayed nonmatching task derives from the fact that prior to testing at the long delays, rats receive a large number of trials at a 4-s delay in order to train the nonmatching rule. After the nonmatching rule is learned, a fixed number of test trials is then given at each of the longer delays. However, as Ringo (1993) pointed out, when more training is given at short delays than at long delays, animals have not been given comparable tests of their ability to perform at these delays. Specifically, the extended training at a 4-s delay, which animals receive while learning the nonmatching rule, might enable them to perform adequately at the 4-s delays, but this training may fail to generalize to longer delays. Such a difficulty could be addressed by giving animals extended testing at long delays until the number of trials they receive matches or exceeds the number of trials they have received at the 4-s delay. If an impairment is still observed at the long delay, then the impairment cannot be due to differential training but must lay in the length of the delay interval itself.

We have evaluated the effects of excitotoxic lesions of the rat hippocampus on the DNMS task with delays from $4 \mathrm{~s}$ to $2 \mathrm{~min}$. All training was postoperative. In the first experiment, after training on the nonmatching rule was completed at a 4-s delay, testing was carried out at progressively longer delays. Four-second probe trials were presented intermittently throughout the course of testing to determine whether animals retained the nonmatching rule. In the second experiment, testing was extended at one of the long delays (1 min) until rats had been given as many 1-min delay trials as 4-s delay trials.

\section{EXPERIMENTAL PROCEDURES}

\section{Subjects}

The subjects were 10 experimentally naive male Long-Evans rats weighing between $300-350 \mathrm{~g}$ at the beginning of the experiment. The animals were randomly assigned to 1 of 2 groups of 5 animals each. The control group (CON) received sham surgery, and the hippocampal group (H-IBO) received bilateral excitotoxic (ibotenic acid) lesions of the dorsal and ventral hippocampus. They were housed individually on a 12:12-h light:dark cycle with continuous access to water.

\section{Surgery}

All surgery was performed using aseptic procedures. Anesthesia was initially induced with an interperitoneal injection of sodium pentobarbital $(65 \mathrm{mg} / \mathrm{kg}$ ip) mixed with $0.2 \mathrm{ml}$ of atropine. The top of the head was shaved and the animal was placed on a heating pad and positioned in a Kopf stereotaxic instrument. Once the animal was in the stereotaxic instrument, anesthesia was maintained throughout the surgery with isoflurane gas $\left(0.8-2.0 \%\right.$ isoflurane delivered in $\mathrm{O}_{2}$ at $\left.1 \mathrm{l} / \mathrm{min}\right)$. The incisor bar was adjusted until bregma was level with lambda. The bone overlying the hippocampus was removed using a sharp bit and high-speed drill.

Ibotenic acid (Biosearch Technologies, San Rafael, CA) was dissolved in $0.1 \mathrm{M}$ phosphate-buffered saline to provide a solution with a concentration of $10 \mathrm{mg} / \mathrm{ml}$ and a $\mathrm{pH}$ of 7.4 . It was injected at a rate of $0.1 \mu \mathrm{l} / \mathrm{min}$ into 18 sites on each side of the brain (modified from Jarrard, 1989; for coordinates, see Clark et al., 2000) using a 10- $\mu$ l Hamilton syringe mounted on a stereotaxic frame and held with a Kopf Microinjector (Model 5000). The syringe needle was first lowered to the surface of the dura. A small puncture was made in the dura just below the needle tip. The syringe needle was then lowered to the target and left in place for 1 min before beginning the injection. The injection volume at each site ranged from $0.05-0.10 \mu \mathrm{l}$, with a total injection volume of $2.04 \mu \mathrm{l}$. Following the injection, the syringe needle was left in place for $2 \mathrm{~min}$ to reduce the spread of IBO up the needle tract. 

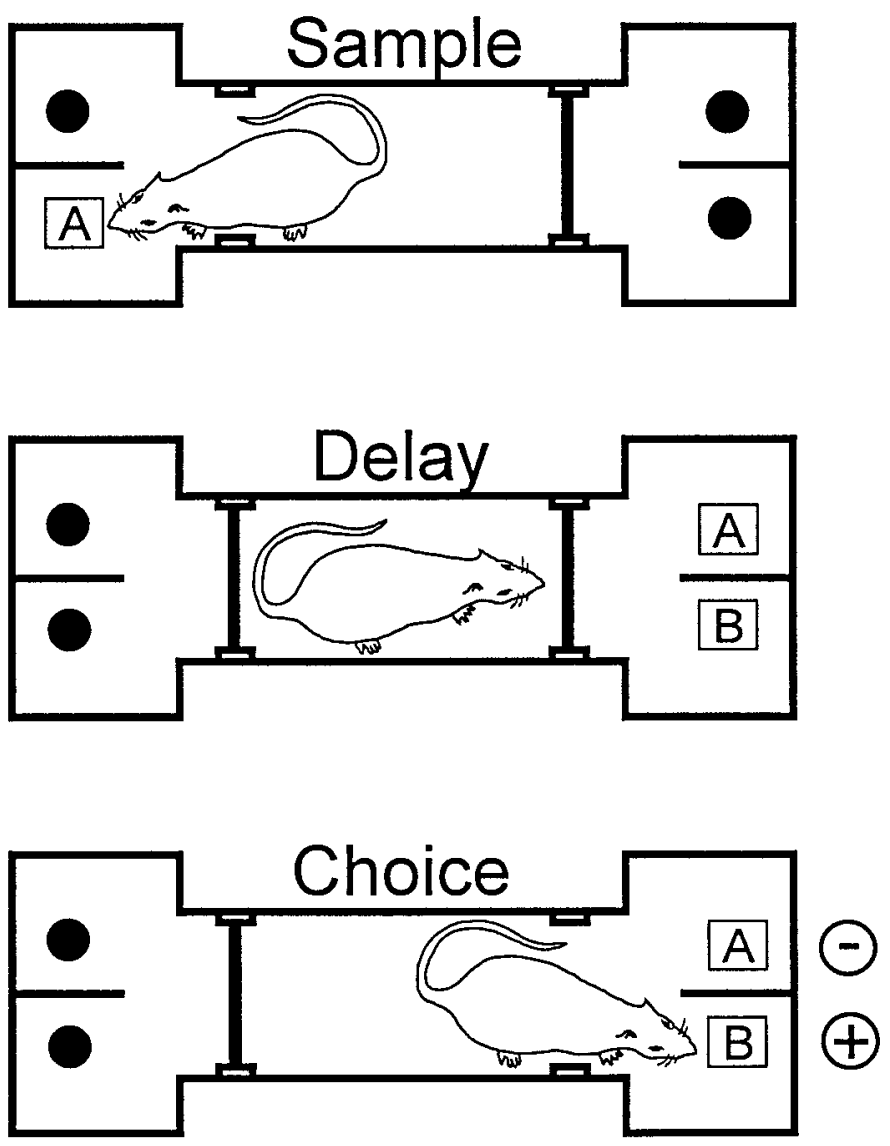

FIGURE 1. Floor plan of apparatus for testing delayed nonmatching-to-sample (DNMS). Goal areas are located at each end of the box. For the sample phase, the rat displaces an object $(A)$ to receive a food reward. In the delay phase, the rat is held in the center of the box by guillotine doors, and two objects ( $A$ and $B$ ) are placed over the food wells. Following the delay interval, the door to the goal area is raised and the rat is allowed to choose between the familiar object (A) and a novel object (B). A reward is delivered to the food well if the rat displaces the novel object $(+)$, but not if the rat displaces the familiar object (-).

After the injections were complete, the wound was closed and the animal was allowed to recover on a heating pad for 3-4 h.

Following the procedure of Mumby et al. (1992), the five animals in the CON group were anesthetized, and placed in the stereotaxic instrument, their heads were shaved, and the skin overlying the area of the hippocampus was opened and then sutured. The CON animals were maintained on isoflurane anesthesia throughout the surgical procedure, just as in the case of the $\mathrm{H}-\mathrm{IBO}$ animals.

\section{Apparatus}

The apparatus was identical in dimensions to the one described by Mumby et al. (1990). It was constructed from sturdy opaque Plexiglas and consisted of a runway that separated two identical goal areas (Fig. 1). Access to the goal areas was controlled by opaque guillotine doors. Each goal area contained two sunken food wells. Sucrose pellets ( $45 \mathrm{mg}$, Noyes Precision Food Pellets, Lancaster, $\mathrm{NH}$ ) could be delivered to the wells by hand through copper tubes that led to the floor of the food wells. The food wells were separated by a small Plexiglas divider $(9 \times 9 \mathrm{~cm})$.

The stimulus set consisted of several hundred objects of various shapes, textures, and colors. Each object was glued to large metal washers that served as a base. Objects were large enough to completely cover the food wells, but small enough to be displaced easily by the rat. Each stimulus object used during the sample phase had a twin that was used during the choice phase (see below) to prevent olfactory cues.

\section{Behavioral Testing}

Following a 2-week recovery from surgery, the $\mathrm{H}-\mathrm{IBO}$ and the $\mathrm{CON}$ rats were placed on a diet until they reached $80 \%$ of their free-feeding weight (approximately 14 days). Subsequent diet adjustments were made daily and were determined by the motivation level of the rats during testing. During this 2-week period, the tester handled each rat for at least 5 min each day. After animals reached their target weight, habituation began with 20 min of exploration in the apparatus each day for 5 consecutive days. During this period, the rats could find food in the wells. During the next 5 days, they learned to approach the doors and move through them when they opened. At this point, the rats quickly moved from one side of the apparatus to the other to find food in the wells.

Behavioral procedures were adapted from the method described by Mumby et al. (1990). Testing was conducted daily for 5 consecutive days each week, approximately $21 \mathrm{~h}$ after the rat's last meal and during the light phase of the light:dark cycle.

\section{Pretraining: Object Discrimination Learning}

Prior to testing on the delayed nonmatching-to-sample task, rats were trained on a two-choice discrimination task to encourage animals to explore and displace objects. The same two objects were used for all the rats. A sucrose pellet was concealed under one of the objects $\left(\mathrm{S}^{+}\right)$, and the rat could obtain the pellet by displacing the object. The other object $\left(S^{-}\right)$was not associated with reward. $S^{+}$was counterbalanced across animals, and the position of $S^{+}$(left/right) varied from trial to trial following a pseudorandom pattern. A trial began when the experimenter opened the door to reveal the two stimulus objects. On the first day, the correct object was baited with food reward at the beginning of the trial. Beginning on the second day, food reward was delivered manually (via a copper tube attached to the food well) when the correct object was displaced. In this way, no odor cues were available at the site of the correct object. The delay between displacement of the object and arrival of food reward in the appropriate food well was less than $1 \mathrm{~s}$. Twenty trials were given each day (ITI $=10 \mathrm{~s}$ ). Training continued until performance reached $80 \%$ correct for 40 consecutive trials (two sessions).

\section{Experiment 1: Delayed Nonmatching to Sample (DNMS)}

\section{Acquisition}

DNMS training began after object discrimination training was completed. During DNMS training, the rat was rewarded for dis- 
placing a sample object (sample phase). Following a 4-s delay, the rat was presented with a choice between an exact copy of the sample object (to avoid odor cues) and a novel object (choice phase). The rat was rewarded for displacing the novel (nonmatching-to-sample) object. For the first two test sessions, the correct object was baited with the food reward at the beginning of the trial to encourage animals to explore and displace objects. After the first two sessions (20 trials), the reward was delivered only after the correct object was displaced. Training continued (10 trials/day) until performance reached $80 \%$ correct over the course of four consecutive test sessions (32/40 correct trials).

For training, 200 objects (and their copies) were grouped in 10 distinct sets of 20 objects (and their copies). In this way, rats saw a given object only once within any 2 -week period, i.e., a different set of 20 objects was used for each session of 10 trials, and different objects were used on each trial of the session (trial unique objects).

\section{Delays}

Following acquisition, the delay between the sample phase and the choice phase was progressively lengthened to $30 \mathrm{~s}, 1 \mathrm{~min}$, and $2 \mathrm{~min}$. Each rat received five consecutive test sessions (10 trials per session, 50 total trials) at each delay. Additionally, during every test session, one 4-s delay trial was administered during the 10-trial test session (in a random position excluding the first and last trial). Thus, 11 trials were given to each rat on each test day. This probe trial made it possible to determine if the rat retained the nonmatching rule as testing proceeded at the longer delays.

\section{Experiment 2: DNMS Extended Training}

After completion of testing on the DNMS task in experiment 1, the CON and $\mathrm{H}-\mathrm{IBO}$ rats were given extended training on the 1-min delay of the DNMS task. As in experiment 1, rats were given 10 trials each day plus a single trial at the 4-s delay. Testing continued until each rat had received 50 trials more at the 1-min delay (in total) than it had received (in total) at the 4-s delay. The total number of trials given at the 1-min delay differed somewhat from rat to rat because rats had originally learned the DNMS task at different rates using the 4-s delay.

\section{Neurohistological Methods}

Rats were administered an overdose of sodium pentobarbital and perfused transcardially with buffered $0.9 \% \mathrm{NaCl}$ solution followed by $10 \%$ formaldehyde solution (in $0.1 \mathrm{M}$ phosphate buffer). Brains were then removed from the skull and cryoprotected in $20 \%$ glycerol $/ 10 \%$ formaldehyde solution. Coronal sections (50 $\mu \mathrm{m})$ were cut with a freezing microtome beginning just anterior to the hippocampus and continuing caudally through the length of the hippocampal region. Every fifth section was mounted and stained with thionin to assess the extent of the lesions.

For each of the H-IBO rats, the extent of damage to the hippocampus was determined by examining under a light microscope each $0.50-\mathrm{mm}$ thionin-stained coronal section through the anteroposterior extent of the hippocampus. The lesions were drawn onto 11 corresponding anteroposterior templates of the normal hip- pocampus, derived from the atlas of Paxinos and Watson (1998). The templates were next "pasted" onto a computer-generated grid of dots. The total number of grid dots contained within the borders of the normal hippocampus, as well as the total number of dots contained within the area of the lesion, was determined for each level. Finally, the total number of grid dots contained within the entire lesion (summing across all of the anteroposterior levels) was divided by the total number of grid dots within the entire normal hippocampus (summing across all of the anteroposterior levels) to determine the percent damage to the hippocampus. The extent of damage to the entorhinal cortex was determined in a similar way.

\section{RESULTS}

\section{Neurohistological Findings}

Figure 2 illustrates the extent of the largest and smallest hippocampal lesion. Figure 3 is a photomicrograph of a representative hippocampal lesion (H3) at four AP levels. All animals had complete, or nearly complete, damage to the CA fields and dentate gyrus. The percent of bilateral damage to the hippocampus averaged 98\% (range, 95-100\%). One animal (H5) had minor sparing of the most medial portion of the anterior aspects of the dorsal hippocampus. Four of the five animals (all except H1) had minor sparing of the most extreme portions of the medial aspect of the ventral hippocampal region, which primarily consists of the dentate gyrus. With respect to extrahippocampal damage, cortical thinning directly above the dorsal hippocampus was present to some extent in all rats. In one rat $(\mathrm{H} 4)$, no damage to the subiculum was detectable. The other four $\mathrm{H}-\mathrm{IBO}$ rats sustained some damage to the ventral subiculum lying adjacent to the ventral hippocampus at more posterior levels. Two rats ( $\mathrm{H} 1$ and $\mathrm{H} 2)$ sustained damage to the entorhinal cortex . The damage in $\mathrm{H} 1$ was bilateral and involved about $70 \%$ of the entorhinal cortex. The damage in $\mathrm{H} 2$ was less extensive, involving only about $32 \%$ of the entorhinal cortex. None of the animals sustained any damage to the perirhinal cortex or the diencephalon.

\section{Behavior}

\section{Pretraining: object discrimination learning}

The groups acquired the object discrimination task in a similar number of trials $(\mathrm{CON}=44.0 \pm 4.0, \mathrm{H}-\mathrm{IBO}=48.0 \pm 8.0$; $\mathrm{t}(8)=0.45, P>0.10)$.

\section{Experiment 1: delayed nonmatching-to-sample (DNMS)}

The two groups learned the DNMS task at the 4-s delay in a similar number of trials $(\mathrm{CON}=220.0 \pm 25.9$; $\mathrm{H}-\mathrm{IBO}=$ $254.0 \pm 37.2 ; \mathrm{t}(8)=0.75, P>0.10$, Fig. 4).

Table 1 and Figure 5 show the performance of the H-IBO and CON animals at each delay. The score for the 4-s delay is the 

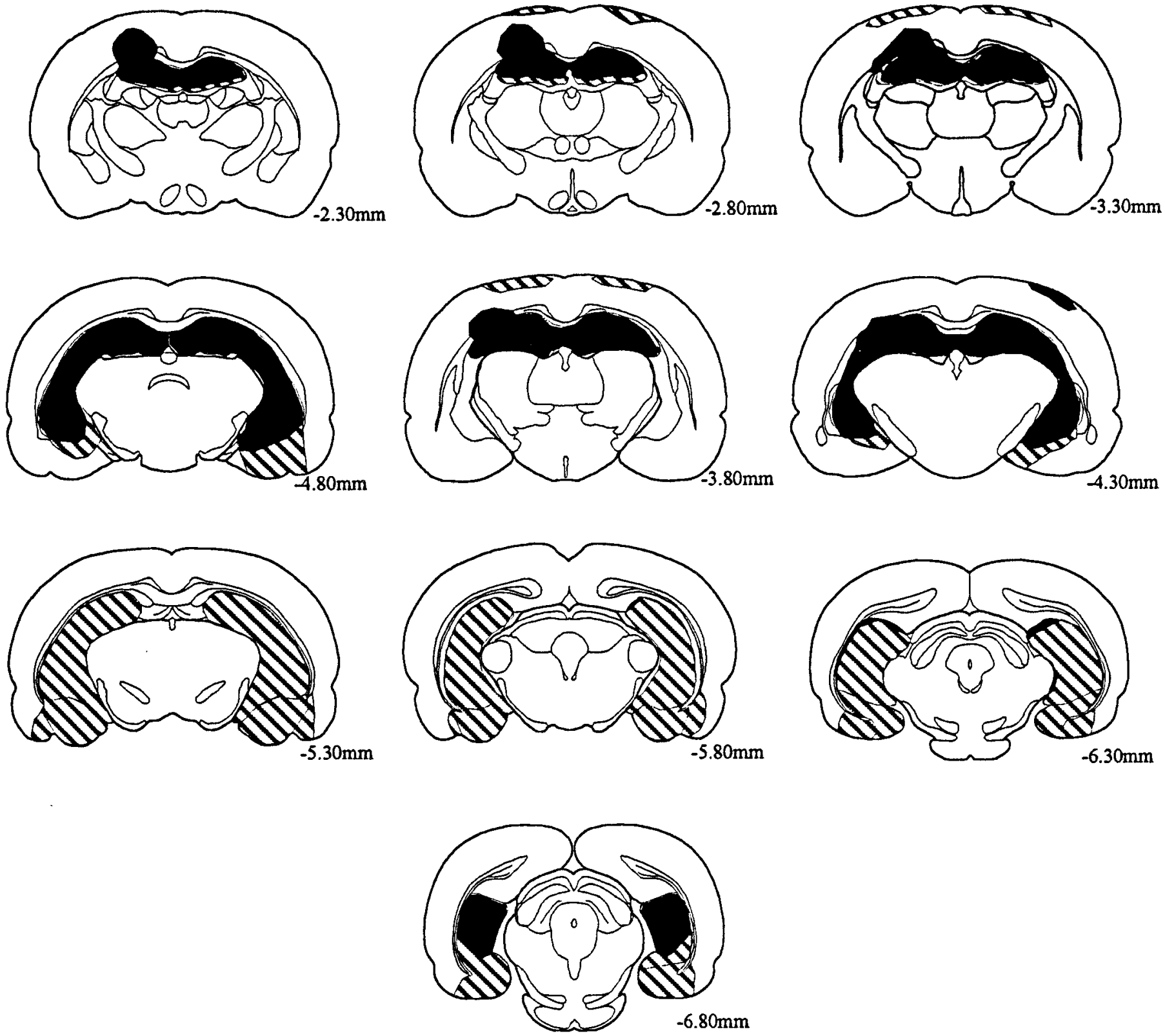

FIGURE 2. Reconstructions of coronal sections through the hippocampus (Paxinos and Watson, 1998) of the largest (striped) and smallest (black) lesion. Numbers represent the distance in millimeters $(\mathrm{mm})$ posterior to from bregma.

percent correct score on the 15 probe trials ( 1 trial per session, 5 trials per delay). The scores for the 30-s, 1-min, and 2-min delays are the percent correct scores on the 50 trials at each delay. A 2 (group) $\times 4$ (delay) repeated measures ANOVA revealed a significant group effect $(\mathrm{F}(1,8)=27.1, P<0.001)$, a significant effect for delay $(\mathrm{F}(1,3)=155.3, P<0.0001)$, and a significant interaction of group $\times$ delay $(\mathrm{F}(1,3)=39.0, P<0.0001)$. Individual planned comparisons revealed that performance on the 4-s probe trials $(\mathrm{t}(8)=0.41, P>0.10)$ and at the 30 -s delay $(\mathrm{t}(8)=0.39$, $P>0.10)$ was not different between the two groups. The H-IBO group was impaired relative to the CON group at the 1 -min $(\mathrm{t}(8)=2.7, P<0.05)$ and 2 -min delays $(\mathrm{t}(8)=12.7, P<$
0.0001). Additionally, the CON group performed significantly above chance at all the delays $(4-\mathrm{s}, \mathrm{t}(4)=18.2, P<0.0001 ; 30$-s, $\mathrm{t}(4)=12.7, P=0.001 ; 1-\min , \mathrm{t}(4)=13.0, P<0.001 ; 2$-min, $\mathrm{t}(4)=15.5 ; P<0.001)$. The H-IBO group performed above chance at the 4-s delay $(\mathrm{t}(4)=34.7, P<0.0001)$, the 30 -s delay $(\mathrm{t}(4)=16.8, P<0.0001)$, and the 1-min delay $(\mathrm{t}(4)=4.4, P<$ $0.05)$, but not at the 2 -min delay $(45.2 \%$ correct).

Finally, the performance of the H-IBO group was further examined at the 1-min and 2-min delays to determine whether performance varied noticeably during the 10 trials of each daily session. The scores for the first 5 trials and the second 5 trials of the test sessions were not measurably different $(P>0.10)$. 

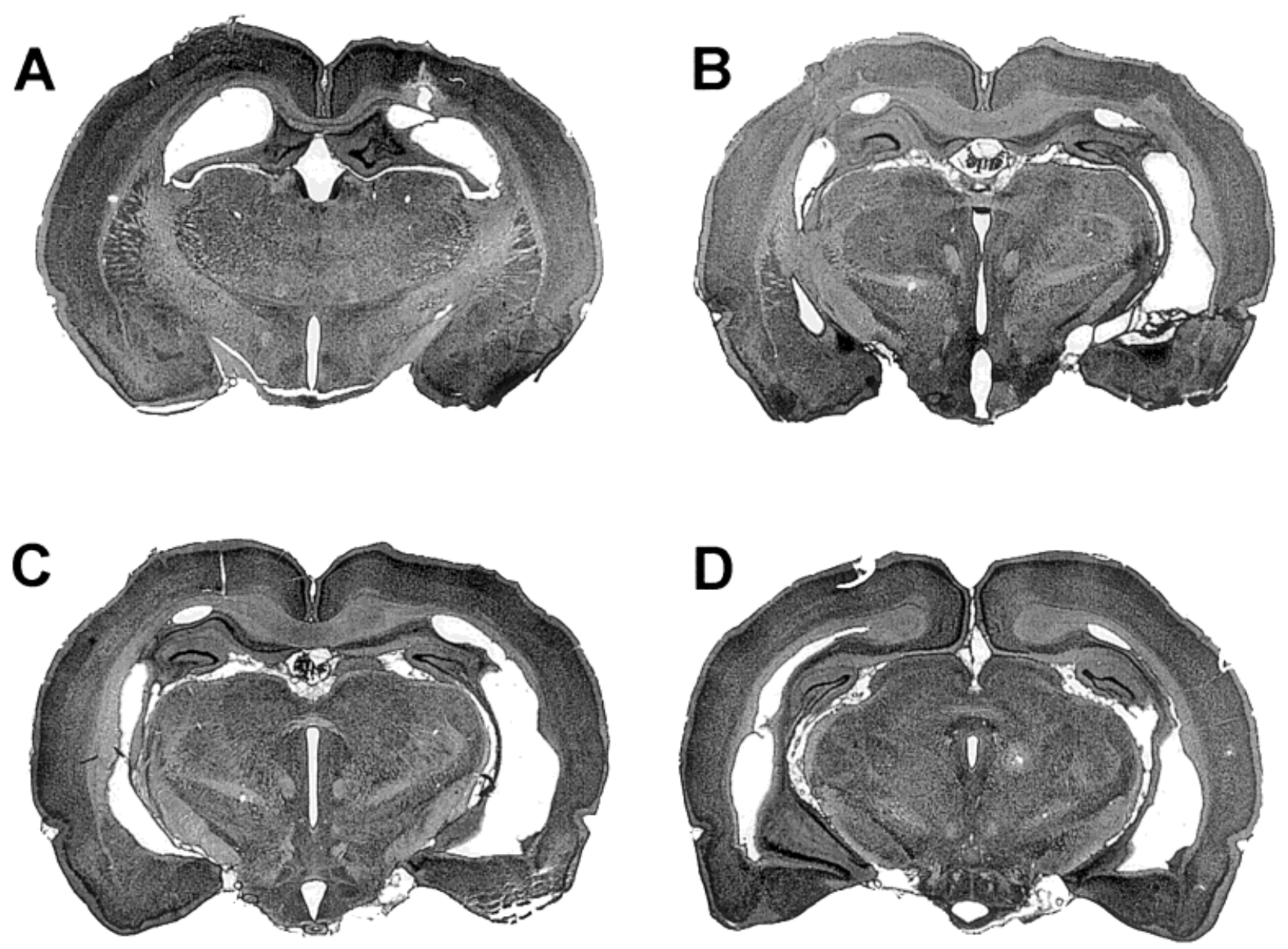

FIGURE 3. Photomicrographs of representative ibotenic acid lesions (rat H3) at four anterior-posterior levels. Sections are arranged from anterior (A) to posterior (D).

\section{Relationship between extent of damage and behavioral scores}

In the $\mathrm{H}-\mathrm{IBO}$ group, two rats ( $\mathrm{H} 1$ and $\mathrm{H} 2)$ sustained some inadvertent damage to the entorhinal cortex, in addition to the intended damage to the hippocampal region. In the case of $\mathrm{H} 1$, this damage was substantial. To evaluate the possible contribution of this damage to the behavioral findings, we performed additional analyses. First, the two rats with damage to the entorhinal cortex and the three rats with lesions of the hippocampus but without damage to the adjacent cortex learned the DNMS task at the 4-s delay in a similar number of trials (mean $=280$ vs. 215 trials, respectively; $P>0.10$ ). Second, performance across delays was virtually identical for the two groups and did not differ by more than $2 \%$ at any delay. Third, performance on the 4-s probe trials was similar for the two groups ( $97 \%$ vs. $100 \%$ for the rats with and without entorhinal damage, respectively). Finally, compared to the five $\mathrm{CON}$ animals, the three rats with hippocampal damage but no damage to the entorhinal cortex were impaired at both the 1-min delay (the three $\mathrm{H}-\mathrm{IBO}$ rats scored $65 \%$ correct; the five $\mathrm{CON}$ rats scored $76 \%$ correct; $P=0.052$ ) and at the 2 -min delay (the three $\mathrm{H}-\mathrm{IBO}$ rats scored $46 \%$ correct; the five CON rats scored $78 \%$ correct; $P<0.0001)$.

\section{Experiment 2: DNMS Extended Training.}

Figure 6 shows performance of the $\mathrm{CON}$ and $\mathrm{H}-\mathrm{IBO}$ groups when they received extended training at a 1-min delay (10 trials/ day) as well as additional 4-s probe trials ( 1 trial/day). Testing continued until each rat had received 50 more trials at the 1-min delay than it had received at the 4-s delay. Thus, the total number of 1-min trials each rat received depended on how quickly it had reached the criterion during the acquisition phase of the DNMS task. At completion of extended training, the CON rats had received an average of 386.8 total trials (range, 300-478) at the 1 -min delay and 336.8 trials (range, 250-428) at the 4-s delay. The H-IBO rats had received an average of 429.8 total trials (range, 267-489) at the 1-min delay and 389.9 trials (range, 217439) at the 4-s delay.

The two groups performed almost identically on the additional 4-s probe trials (Fig. 6; $\mathrm{CON}=95.2 \% \pm 1.2 \% ; \mathrm{H}-\mathrm{IBO}=92.6 \%$ $\pm 1.2 \% ; \mathrm{t}(8)=1.6, P>0.10)$. At the 1 -min delay, the H-IBO group was markedly impaired relative to the $\mathrm{CON}$ group $(\mathrm{CON}=$ $81.2 \% \pm 2.6 \% ; \mathrm{H}-\mathrm{IBO}=52.2 \% \pm 1.0 \% ; \mathrm{t}(8)=10.3, P<$ 0.0001). The performance of the CON group was well above chance $(\mathrm{t}(4)=11.8, P<0.001)$, whereas the performance of the $\mathrm{H}-\mathrm{IBO}$ group was not $(\mathrm{t}(4)=2.2, P=0.097)$. It is also notewor- 


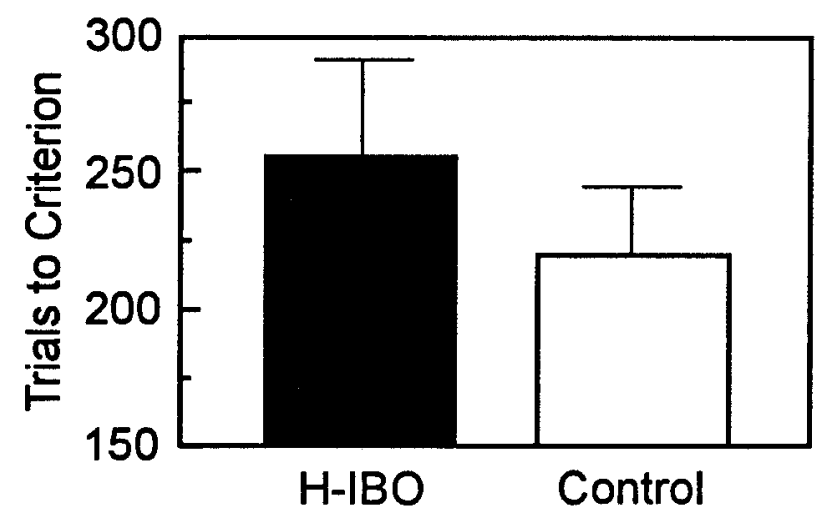

FIGURE 4. Mean number of trials to reach criterion on the delayed nonmatching-to-sample task for the control group $(n=5)$ and the H-IBO group $(n=5)$. A 4-s delay interval was used for training. There was no difference between groups. Brackets indicate SEM.

thy that extended training at the 1-min delay marginally improved the performance of the CON group, relative to the performance of this group on the 50 test trials that had already been given at the 1 -min delay (Figs. 5, 6; 81.2\% vs. $76.0 \%, \mathrm{t}(4)=2.5, P=0.067$ ). In contrast, for the $\mathrm{H}-\mathrm{IBO}$ group, extended training at the 1 -min delay resulted in somewhat poorer performance (Figs. 5, 6; 52.2\% vs. $65.2 \%, \mathrm{t}(4)=4.3, P<0.05)$. There was also a significant interaction of training condition (experiment 1 vs. experiment 2) and lesion group $(\mathrm{F}(1)=13.9, P<0.001)$. Finally, performance on the 4-s delay trials that were given during extended training was a little lower than what it had been during the initial probe trials (CON group, $95.2 \%$ vs. $97.4 \% \mathrm{t}(4)=0.85, P>0.10$; H-IBO group, $92.6 \%$ vs. $98.7 \%$; $\mathrm{t}(4)=3.2, P<0.05)$.

TABLE 1.

Performance on DNMS

\begin{tabular}{lrrcr}
\hline Delays & \multicolumn{3}{l}{} \\
\hline Rat & \multicolumn{1}{c}{$4 \mathrm{~s}$} & $30 \mathrm{~s}$ & $1 \mathrm{~min}$ & $2 \mathrm{~min}$ \\
\hline H1 & 93.0 & 76.0 & 72.0 & 42.0 \\
H2 & 100.0 & 70.0 & 58.0 & 46.0 \\
H3 & 100.0 & 76.0 & 72.0 & 52.0 \\
H4 & 100.0 & 70.0 & 68.0 & 44.0 \\
H5 & 100.0 & 72.0 & 56.0 & 42.0 \\
Mean & 98.6 & 72.8 & 65.2 & 45.2 \\
C1 & 100.0 & 74.0 & 76.0 & 78.0 \\
C2 & 100.0 & 82.0 & 82.0 & 82.0 \\
C3 & 100.0 & 70.0 & 78.0 & 82.0 \\
C4 & 100.0 & 74.0 & 74.0 & 78.0 \\
C5 & 87.0 & 74.0 & 70.0 & 72.0 \\
Mean & 97.4 & 74.8 & 76.0 & 78.4 \\
\hline
\end{tabular}

Percent correct score on the delayed nonmatching-to-sample task for the Control group (C1-C5) and the H-IBO group (H1-H5) across delays. The score for the 4-sec delay was the score for 154 -sec probe trials that were presented during the course of testing at the $30-\mathrm{sec}, 1 \mathrm{~min}$, and 2-min delays (50 trials/delay).

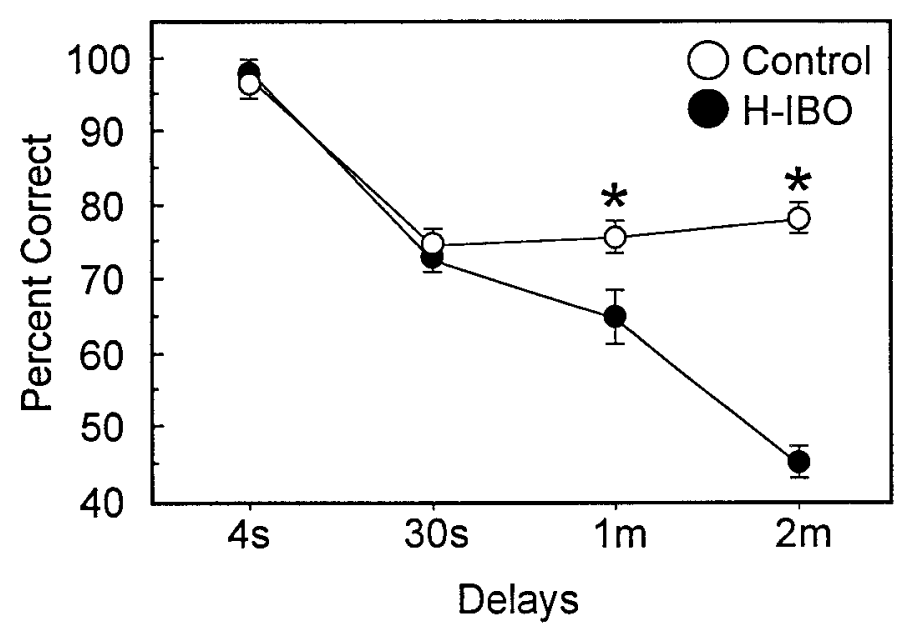

FIGURE 5. Percent correct score on the delayed nonmatchingto-sample task for the control group (open circles, $n=5$ ) and the H-IBO group (solid circles, $n=5$ ) across delays. The score for the 4-s delay was the score for 15 4-s probe trials that were presented during the course of testing at the 30-s, 1-min, and 2-min delays (50 trials/ delay). Asterisks indicate impaired performance of the H-IBO group relative to the control group $(P<0.05)$. Brackets indicate SEM.

\section{Relationship between extent of damage and behavioral scores}

To evaluate the possible contribution of inadvertent damage to the entorhinal cortex in rats $\mathrm{H}-\mathrm{IBO} 1$ and $\mathrm{H}-\mathrm{IBO} 2$, we performed additional analyses. First, the two rats that sustained damage to the entorhinal cortex and the three rats with lesions of the hippocampus but without damage to the adjacent cortex performed similarly at the 4 -s delay (33 trials, 94\% correct vs. 41 trials, $92 \%$ correct, respectively; $P>0.10)$. The two groups also performed similarly

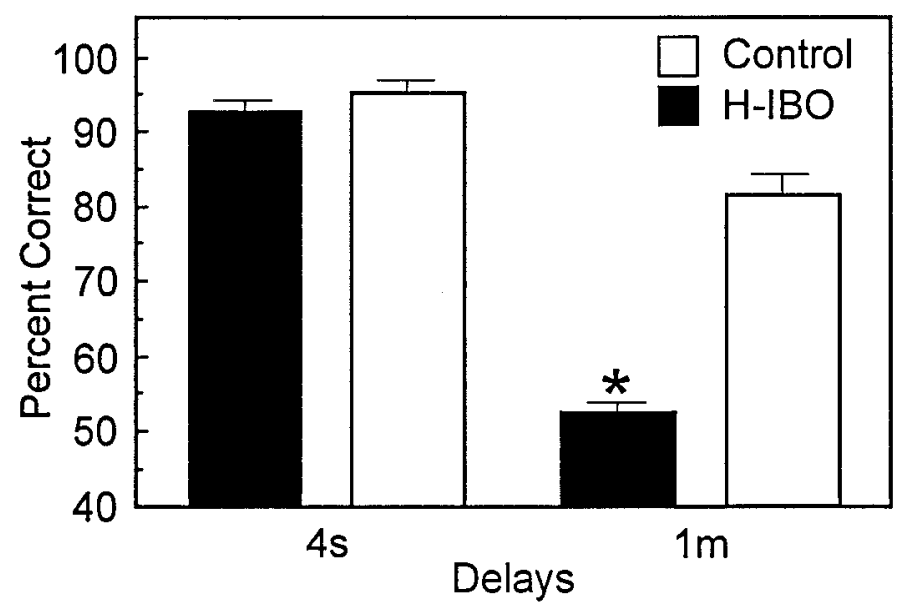

FIGURE 6. Percent correct score for the control $(n=5)$ and H-IBO $(n=5)$ groups when extended training was given at the 1 -min delay (10 trials/day) and additional 4-s probe trials ( 1 trial/day) were presented. When extended training was completed, rats had received more training at the 1-min delay than at the 4-s delay. Asterisk indicates impaired performance of the $\mathrm{H}$-IBO group relative to the control group $(P<.01)$. Brackets indicate SEM. 
during the extended training at the 1-min delay (328 trials, 52\% correct vs. 406 trials, $53 \%$ correct; $P>0.10$ ). Finally, compared to the five CON animals, the three rats with hippocampal damage but no damage to the entorhinal cortex were impaired during extended training at the 1-min delay trials (the three $\mathrm{H}$-IBO rats scored 53\% correct; the five CON rats scored 81\% correct; $P<$ $0.0001)$.

\section{DISCUSSION}

The results of experiment 1 show that the hippocampus is critical for normal recognition memory. Rats with hippocampal lesions learned the nonmatching rule for the DNMS task as quickly as the control group. However, they performed more poorly than the control group when the delay between the sample and choice phase was increased to 1 or $2 \mathrm{~min}$. An impairment was observed at these longer delays, despite the fact that performance was normal on 4-s delay trials that occurred daily in the same sessions as the long-delay trials. Intact performance on these 4-s probe trials shows that animals with hippocampal lesions retained the nonmatching rule throughout testing. In addition, normal performance at the 4-s delay shows that impaired perception, attention, or motivation is unlikely to account for the findings. The trials did not differ from each other during the first few seconds, and no information was available at the outset to distinguish a 4-s delay trial from a longer delay trial. Yet, animals were able to remember the sample object for $4 \mathrm{~s}$ and failed only when they were required to remember the sample object for $1 \mathrm{~min}$ or longer. These findings show that the impairment following hippocampal lesions in rats was delay-dependent and suggest that the impairment occurred because of the greater demands placed on memory at longer delays. Insofar as we could determine, there was no suggestion that inadvertent damage to the entorhinal cortex contributed to the impaired performance of the $\mathrm{H}$-IBO group.

This contrast between intact performance at short delays and impaired performance at long delays by the $\mathrm{H}-\mathrm{IBO}$ animals was further illuminated by giving extended training on the DNMS task at a delay of $1 \mathrm{~min}$ (experiment 2). It had been pointed out previously that performance in the DNMS task might be related less to the delay than to how much training is given at each delay (Ringo, 1993). Ordinarily, animals receive many more trials at the short delay that is used to train the nonmatching rule than at any other delay. In our study, the extended training served to give animals even more practice at the 1-min delay than they had been given at the 4-s delay. Nevertheless, despite the extended training, the performance of rats with hippocampal lesions remained impaired at the long delay and remained intact at the short (4-s) delay. For the $\mathrm{H}-\mathrm{IBO}$ animals, the impairment during extended training at the 1 -min delay (experiment 2) was greater than during the 50 test trials of experiment $1(52.2 \%$ vs. $65.2 \%$ correct). This finding is likely due to the fact that the extended training at the 1-min delay occurred immediately after testing had been concluded at the 2-min delay (experiment 1 ). The average score for the H-IBO animals at the 2-min delay was only $45.2 \%$ correct, and perhaps after many trials of poor performance, there was a reduction in motivation at long delays. In any case, these results indicate that extended training at long delays does not result in good performance.

At least three important issues need to be considered when using the DNMS task to evaluate recognition memory following hippocampal lesions. First, the DNMS task appears to be more sensitive to hippocampal damage when no pretraining is administered. For example, when preoperative training was given, Mumby et al. (1992) reported that hippocampal lesions impaired performance at a 10 -min delay, but not at a 2 -min delay. When no preoperative training was given, hippocampal lesions impaired performance at delays of 2 min (Mumby et al., 1995). In our study, no preoperative training was given, and an impairment in rats with hippocampal lesions was observed at a delay as short as $1 \mathrm{~min}$. Preoperative training may provide animals with extended practice at holding novel objects in memory across short delays, which might then make it easier to hold novel objects in memory across longer delays (Bachevalier et al., 1999; Ringo, 1993; Zola et al., 2000).

Second, sufficiently long delay intervals are needed to reveal memory impairment. In our study, a 30-s delay was not sufficient to detect an impairment; an impairment emerged only at delays of 1 or $2 \mathrm{~min}$. Consistent with this finding, no impairment was found following hippocampal lesions in studies that used maximum delay intervals of $30 \mathrm{~s}$ or less (Rothblat and Kromer, 1991; Kesner et al., 1993). Apparently, the demand on memory must be sufficiently great to detect memory impairment following hippocampal lesions. At the same time, the retention interval needed to reveal a memory impairment after hippocampal lesions can be expected to vary across species and across tasks. In addition, the chance of detecting an impairment at a given retention interval may be improved by testing larger numbers of animals. Thus, an early study of four monkeys with hippocampal lesions found significantly impaired performance on the DNMS task at retention intervals of 10 min and $40 \mathrm{~min}$ (Alvarez et al., 1995). When data became available from 14 additional monkeys with hippocampal lesions, a deficit could be detected in all 18 animals at a retention interval as short as $15 \mathrm{~s}$ (Zola et al., 2000).

Finally, if recognition memory is to be impaired, a relatively large portion of the hippocampus may need to be damaged. Dorsal hippocampal lesions involving as little as $20-30 \%$ of total hippocampal volume are sufficient to impair spatial memory (Moser et al., 1995). Consistent with this finding, Duva et al. (1997) found that small dorsal lesions of the hippocampus impaired spatial memory. However, performance on the DNMS task was not affected, even when the delay was extended to $5 \mathrm{~min}$. We suggest that the hippocampus is important for both spatial memory and object recognition memory, but that spatial memory tasks are more sensitive to hippocampal damage than are recognition tasks.

An additional consideration related to lesion size involves hippocampal lesions that are induced by ischemia. Ischemic damage to the hippocampus clearly produces an impairment on the DNMS task in both monkeys (Zola-Morgan et al., 1992) and rats (Wood et al., 1993; Mumby et al., 1996). These findings seemingly provide support for the critical involvement of the hippocam- 
pus in recognition memory. However, it has been suggested that this impairment is due to secondary or covert damage to structures outside of the hippocampus (e.g., Bachevalier and Mishkin, 1989; Wood et al., 1993; but see Squire and Zola, 1996). In the only study to examine this possibility directly (Mumby et al., 1996), it was reported that ischemia significantly reduced postoperative scores on the DNMS task relative to preoperative scores. Yet, ischemia plus aspiration/electrolytic lesions of the hippocampus did not significantly reduce postoperative scores. Mumby et al. (1996) suggested that hippocampal lesions prevented the ischemia from damaging structures outside the hippocampus. However, to substantiate this interpretation, the postoperative scores of the ischemia-only group should have been lower than the postoperative scores of the ischemia plus hippocampal lesion group. This comparison was not reported, and Figure 5 of that study casts doubt as to whether there is a measurable difference between these scores. It is also worth noting that monkeys with ischemic lesions of the hippocampus exhibited a level of impairment on the DNMS task that is similar to the level of impairment exhibited by monkeys with either ibotenic acid or radio-frequency lesions of the hippocampus (Zola-Morgan et al., 1994; Zola et al., 2000) and an impairment that is less severe than that exhibited by monkeys with large lesions of the medial temporal lobe (Zola-Morgan et al., 1994). The nature of the behavioral impairment that results from ischemic damage is an important question that warrants further investigation.

Several studies have evaluated recognition memory in rats following hippocampal damage, using tasks similar to the DNMS task that we used. In one study, rats with hippocampal lesions were unimpaired on a continuous object recognition task that measured the increase in latency to displace objects that were repeated and not reinforced (Jackson-Smith et al., 1993). However, the longest delay tested corresponded to the length of the intertrial interval, which was only $10 \mathrm{~s}$. It is possible that rats with hippocampal damage would be impaired on this task if the delay were as long as 1 or $2 \mathrm{~min}$. In another study, Aggleton et al. (1986) assessed nonmatching-to-sample performance using a Y-maze with detachable arms that contained trial-unique objects. Rats with aspiration lesions of the hippocampus were unimpaired at all delays, even when the delay was extended to $60 \mathrm{~s}$. However, the rats in this study appeared to have less hippocampal damage than the rats in the present study. Moreover, it is possible that an impairment might have been observed had the delay interval been increased to $2 \mathrm{~min}$ (both groups were well above chance at the 1-min delay).

In a third study, Steele and Rawlins (1993) also used a Y-maze and a continuous nonmatching-to-sample task with objects. In this study, rats with hippocampal damage were impaired relative to the control group in all task conditions. The easiest task condition involved an interval between sample and choice of $24 \mathrm{~s}$. Although a delay of $24 s$ was not sufficient to find an impairment in some of the studies reviewed here, a delay of this length is sufficient to reveal impaired recognition memory in both humans (Buffalo et al., 1998) and monkeys (Zola et al., 2000) with hippocampal damage.

Another test of recognition memory that appears to have memory requirements similar to the requirements of the DNMS task is the visual paired-comparison (or spontaneous nonmatching-tosample) task. In the version developed for humans and monkeys, subjects are first presented with two identical stimuli and given time to look at them. When one of the familiar stimuli is subsequently presented together with a novel stimulus, normal subjects spend more time looking at the novel stimulus than at the familiar stimulus. This preference for the novel stimulus indicates a memory of the familiar and now less interesting stimulus. Both humans (McKee and Squire, 1993) and monkeys (Bachevalier et al., 1993; Pascalis and Bachevalier, 1999; Zola et al., 2000) with damage to the hippocampus exhibit a delay-dependent memory impairment on this task. A rodent version of this task has also been developed in which animals first explore two identical objects in an open arena. Subsequently, a novel object and one of the familiar objects are presented together, and memory for the previously presented object is reflected in the fact that the animal spends more time exploring the novel object than the familiar object (Ennaceur and Delacour, 1988). Direct damage to the hippocampus by ibotenic acid results in a delay-dependent memory impairment on this task in rats (Clark et al., 2000). In contrast, fornix lesions have been reported to spare performance on this task (Ennaceur and Aggleton, 1994; Ennaceur et al., 1996, 1997; Clark, 2000). The idea that fornix lesions are not equivalent to direct damage to the hippocampus is also supported by the finding that selective hippocampal lesions produce more profound spatial memory impairment as well as larger increases in locomotor activity than do lesions of the fimbria-fornix (Cassel et al., 1998).

Impaired recognition memory following restricted hippocampal damage has been well-documented in humans and in monkeys. In humans, circumscribed hippocampal damage has been confirmed in postmortem examination in four cases (Zola-Morgan et al., 1986; Rempel-Clower et al., 1996). All four patients had impaired recognition memory. In monkeys with damage limited to the hippocampus, recognition memory was also impaired on both the DNMS task (Zola-Morgan et al., 1992; Zola et al., 2000; Alvarez et al., 1995; Beason-Held et al., 1999) and on the visual paired-comparison task (Pascalis and Bachevalier, 1999; Zola et al., 2000).

The present results extend this same pattern of finding to the rat. Recently, impaired recognition memory has also been described in mice lacking the NMDA-R1subunit in the CA1 region of the hippocampus (Rampon et al., 2000). It is interesting to consider why it has been difficult to reach a consensus regarding the importance of the rodent hippocampus for recognition memory. One factor could involve the three methodological issues discussed above, which might influence what behavioral findings are obtained: 1) possible mitigating effect of preoperative training, 2) length of delay, and 3) size of hippocampal lesion. A second factor may be related to the difficulty in interpreting rodent performance as evidence for a delay-dependent memory deficit. In the present study, this difficulty was addressed by the use of 4-s probe trials and by giving extended training at one of the longer delays (for another way that this difficulty has been addressed in studies with monkeys, see Alvarez et al., 1994).

Finally, a third factor may be related to the robust impairments in spatial memory that have been widely reported in rats with 
hippocampal lesions. Impaired recognition memory is not as readily observed after hippocampal lesions as is impaired spatial memory (Duva et al. 1997). One interpretation of this finding is that the hippocampus is specialized for processing spatial/temporal contextual information, and other structures outside of the hippocampus can accomplish familiarity-based judgments of recognition (Aggleton and Brown, 1999). Another possibility is that spatial memory tasks are especially sensitive to the integrity of the hippocampus. For example, spatial memory tasks have much in common with tasks of free recall, because usually an arbitrary location (e.g., a platform submerged in water) must be recalled from memory. Moreover, it is well-documented that tasks of free recall are more difficult and more sensitive to memory impairment than are tasks of recognition memory (Haist et al., 1992). Thus, spatial memory tasks might be viewed as assessing the same memory function that can be assessed as well by other tasks that depend on nonspatial memory abilities (e.g., olfactory memory; Bunsey and Eichenbaum, 1996) and by tasks like the DNMS that measure the capacity for recognition of familiarity. A similar view has been outlined in detail by Eichenbaum (1999). In this view, both the ability to recognize an object as familiar and spatial memory tasks depend on the ability to relate elements within a context.

In summary, a recognition judgment requires that the stimulus presented in the retention test be identified as what was presented during the learning. Thus, in the case of recognition, an association must be made between the object and the context in which it was presented. It is this ability to form relationships and conjunctions among stimuli that we suggest is at the heart of hippocampus-dependent (declarative) memory in humans, monkeys, and rodents.

\section{Acknowledgments}

We thank Len Jarrard for providing coordinates for ibotenic acid lesions, Tony Phillips, John Pinel, and Tom Kornecook for help with the nonmatching-to-sample task, Cecelia and Jeff Manzanares for constructing the apparatus, and Lisa Stefanacci for assistance with neurohistological analysis.

\section{REFERENCES}

Aggleton JP, Brown MW. 1999. Episodic memory, amnesia, and the hippocampal-anterior thalamic axis. Behav Brain Sci 22:425-444.

Aggleton JP, Hunt, PR, Rawlins JNP. 1986. The effects of hippocampal lesions upon spatial and non-spatial tests of working memory. Behav Brain Res 19:133-146.

Alvarez P, Zola-Morgan S, Squire LR. 1994. The animal model of human amnesia: long-term memory impaired and short-term memory intact. Proc Natl Acad Sci USA 91:5637-5641.

Alvarez P, Zola-Morgan SM, Squire LR. 1995. Damage limited to the hippocampal region produces long-lasting memory impairment in monkeys. J Neurosci 15:3796-3807.

Bachevalier J, Meunier M. 1996. Cerebral ischemia: are the memory deficits associated with hippocampal cell loss? Hippocampus 6:553-560.

Bachevalier J, Mishkin M. 1989. Mnemonic and neuropathological effects of occluding the posterior cerebral artery in Macaca mulatta. Neuropsychologia 27:83-105.
Bachevalier J, Brickson M, Hagger C. 1993. Limbic-dependent recognition memory in monkeys develops early in infancy. Neuroreport 4:77-80.

Bachevalier J, Beauregard M, Alvarado MC. 1999. Long-term effects of neonatal damage to the hippocampal formation and amygdaloid complex on object discrimination and object recognition in rhesus monkeys (Macaca mulatta). Behav Neurosci 113:1127-1151.

Beason-Held LL, Rosene DL, Killiany RJ, Moss MB. 1999. Hippocampal formation lesions produce memory impairment in the rhesus monkey. Hippocampus 9:562-574.

Buffalo EA, Reber PJ, Squire LR. 1998. The human perirhinal cortex and recognition memory. Hippocampus 8:330-339.

Bunsey M, Eichenbaum H. 1996. Conservation of hippocampal memory function in rats and humans. Nature 379:255-257.

Cassel J, Cassel S, Galani R, Kelche C, Will B, Jarrard L. 1998. Fimbria-fornix vs. selective hippocampal lesions in rats: effects on locomotor activity and spatial learning and memory. Neurobiol Learn Mem 69:22-45.

Clark RE, Zola SM, Squire LR. 2000. Impaired recognition memory in rats after damage to the hippocmapus. J Neurosci 20:8853-8860.

Duva CA, Floresco SB, Wunderlich GR, Lao TL, Pinel JPJ, Phillips AG. 1997. Disruption of spatial but not object-recognition memory by neurotoxic lesions of the dorsal hippocampus in rats. Behav Neurosci 111:1184-1196.

Eichenbaum H. 1997. Declarative memory: insights from cognitive psychology. Annu Rev Psychol 48:547-572.

Eichenbaum H. 1999. The hippocampus and mechanisms of declarative memory. Behav Brain Res 103:123-133.

Ennaceur A, Aggleton JP. 1994. Spontaneous recognition of object configurations in rats: effects of fornix lesions. Exp Brain Res 100:85-92.

Ennaceur A, Delacour J. 1988. A new one-trial test for neurobiological studies of memory in rats: 1 . Behavioural data. Behav Brain Res 31: 47-59.

Ennaceur A, Neave N, Aggleton JP. 1996. Neurotoxic lesions of the perirhinal cortex do not mimic the behavioural effects of fornix transection in the rat. Behav Brain Res 80:9-25.

Ennaceur A, Neave N, Aggleton JP. 1997. The effects of neurotoxic lesions of the perirhinal cortex combined to fornix transection on object recognition memory in the rat. Behav Brain Res 88:181-193.

Haist F, Shimamora AP, Squire LR. 1992. On the relationship between recall and recognition memory. J Exp Psychol [Learn Mem Cogn] 18:691-702.

Jackson-Smith P, Kesner RP, Chiba AA. 1993. Continuous recognition of spatial and nonspatial stimuli in hippocampal-lesioned rats. Behav Neural Biol 59:107-119.

Jarrard LE. 1989. On the use of ibotenic acid to lesion selectively different components of the hippocampal formation. J Neurosci Methods 29: 251-259.

Kesner RP, Bolland BL, Dakis M. 1993. Memory for spatial location, motor responses, and objects: triple dissociation among the hippocampus, caudate nucleus, and extrastriate visual cortex. Exp Brain Res 93:462-470.

Manns JR, Squire LR. 1999. Impaired recognition memory on the Doors and People Test after damage limited to the hippocampal region. Hippocampus 9:495-499.

McKee RD, Squire LR. 1993. On the development of declarative memory. J Exp Psychol [Learn Mem Cogn] 19:397-404.

Milner B. 1972. Disorders of learning and memory after temporal lobe lesions in man. Clin Neurosurg 19:421-46.

Moser MB, Moser EI, Forrest E, Anderson P, Morris RG. 1995. Spatial learning with a minislab in the dorsal hippocampus. Proc Natl Acad Sci USA 92:9697-9701.

Mumby DG, Pinel JPJ, Wood ER. 1990. Nonrecurring-items delayed nonmatching-to-sample in rats: a new paradigm for testing nonspatial working memory. Psychobiology 18:321-326.

Mumby DG, Wood ER, Pinel JPJ. 1992. Object-recognition memory is only mildly impaired in rats with lesions of the hippocampus and amygdala. Psychobiology 20:18-27. 
Mumby DG, Pinel JPJ, Kornecook TJ, Shen MJ, Redila VA. 1995. Memory deficits following lesions of hippocampus or amygdala in rat: assessment by an object-memory test battery. Psychobiology $23: 26-36$

Mumby DG, Wood ER, Duva CA, Kornecook TJ, Pinel JPJ, Phillips AG. 1996. Ischemia-induced object-recognition deficits in rats are attenuated by hippocampal ablation before or soon after ischemia. Behav Neurosci 110:266-281.

Murray EA, Mishkin M. 1998. Object recognition and location memory in monkeys with excitotoxic lesions of the amygdala and hippocampus. J Neurosci 18:6568-6582.

Pascalis OP, Bachevalier J. 1999. Neonatal aspiration lesions of the hippocampal formation impair visual recognition memory when assessed by paired-comparison task but not by delayed nonmatching-to-sample task. Hippocampus 9:609-616.

Paxinos G, Watson C. 1998. The rat brain in stereotaxic coordinates, 4th ed. San Diego, CA: Academic Press.

Rampon C, Ya-Ping T, Goodhouse J, Shimizu E, Kyin M, Tsien JZ. 2000. Enrichment induces structural changes and recovery from nonspatial memory deficits in CA1 NMDAR1-knockout mice. Nat Neurosci 3:238-244.

Reed JM, Squire LR. 1997. Impaired recognition memory in patients with lesions limited to the hippocampal formation. Behav Neurosci 111: 667-675.

Rempel-Clower N, Zola SM, Squire LR. 1996. Three cases of enduring memory impairment following bilateral damage limited to the hippocampal formation. J Neurosci 16:5233-5255.

Ringo JL. 1993. Spared short-term memory in monkeys following medial temporal lobe lesions is not yet established: a reply to Alvarez-Royo, Zola-Morgan and Squire. Behav Brain Res 59:65-72.
Rothblat LA, Kromer LR. 1991. Object recognition memory in the rat: the role of the hippocampus. Behav Brain Res 42:25-32.

Squire LR. 1992. Memory and the hippocampus: a synthesis from findings with rats, monkeys and humans. Psychol Rev 99:195-231.

Squire LR, Zola SM. 1996. Ischemic brain damage and memory impairment: a commentary. Hippocampus 6:546-552.

Steele K, Rawlins JNP. 1993. The effects of hippocampectomy on performance by rats on a running recognition task using long list of nonspatial items. Behav Brain Res 54:1-10.

Wiig KA, Bilkey DK. 1995. Lesions of rat perirhinal cortex exacerbate the memory deficit observed following damage to the fimbria-fornix. Behav Neurosci 109:620-630.

Wood ER, Mumby DG, Pinel JPJ, Phillips AG. 1993. Impaired object recognition memory in rats following ischemia-induced damage to the hippocampus. Behav Neurosci 107:51-62.

Zola SM, Squire LR, Teng E, Stefanacci L, Buffalo EA, Clark RE. 2000. Impaired recognition memory in monkeys after damage limited to the hippocampal region. J Neurosci 20:451-463.

Zola-Morgan SM, Squire LR. 1993. Neuroanatomy of memory. Annu Rev Neurosci 16:547-563.

Zola-Morgan SM, Squire LR, Amaral DG. 1986. Human amnesia and the medial temporal region: enduring memory impairment following a bilateral lesion limited to field CA1 of the hippocampus. J Neurosci 6:2950-2967.

Zola-Morgan SM, Squire LR, Rempel NL, Clower RP, Amaral DG. 1992. Enduring memory impairment in monkeys after ischemic damage to the hippocampus. J Neurosci 9:4355-4370.

Zola-Morgan SM, Squire LR, Ramus SJ. 1994. Severity of memory impairment in monkeys as a function of locus and extent of damage within the medial temporal lobe memory system. Hippocampus 4:483-495. 